\title{
BIOMINERALS
}

\section{Tomography reveals all}

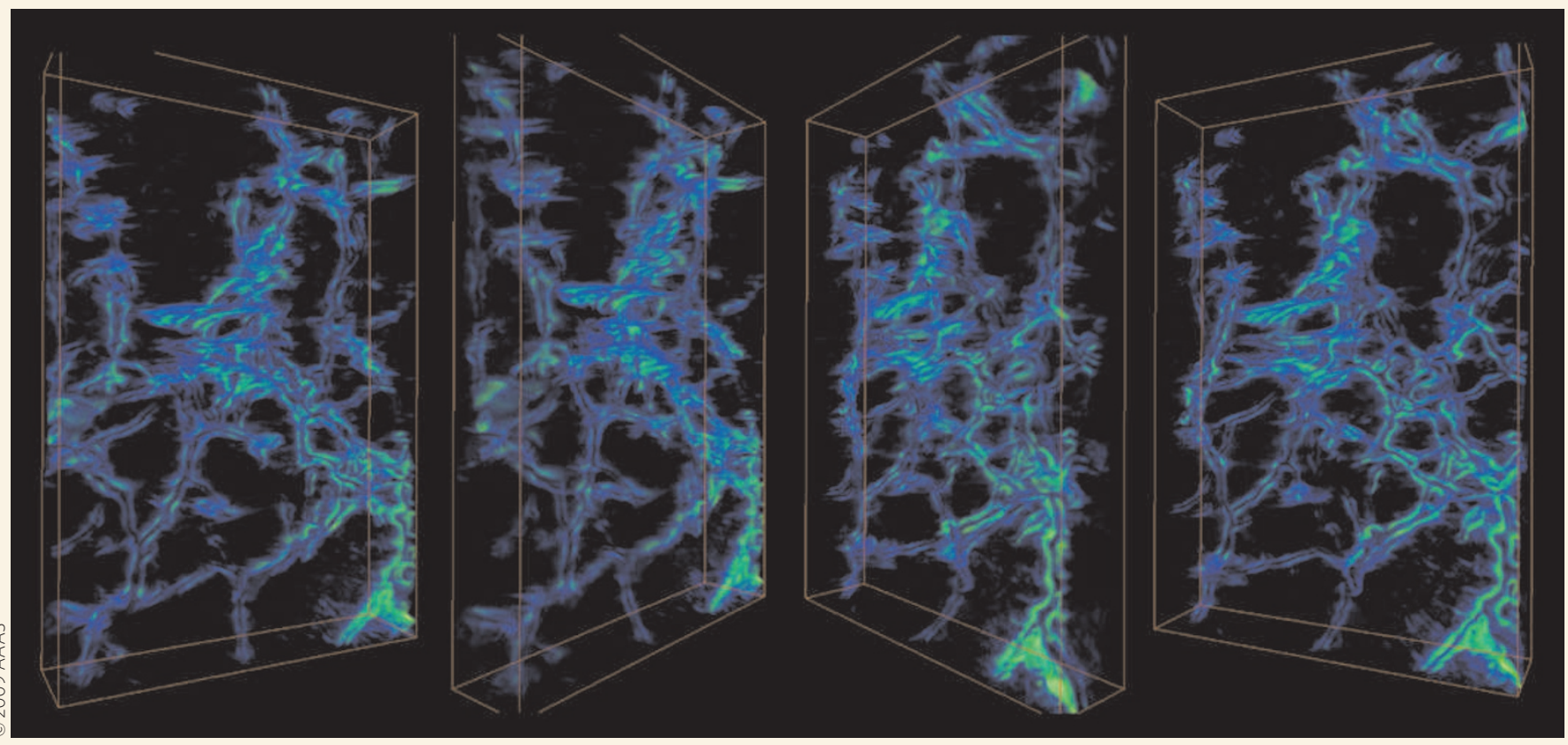

Biominerals, such as sea urchin spines or mollusc shells, are sources of inspiration and envy to materials researchers. These inorganic-organic hybrids have exceptional mechanical properties, such as strength, thanks to the intricate way the two components are arranged. Although methods of investigating how the biopolymers are distributed through the inorganic crystal lattice have been developed, none is completely satisfactory and they yield little information about the interface between polymer and crystal.

Now, Lara Estroff and colleagues from Cornell University have used electron tomography to image, in three dimensions, the internal structure of calcite single crystals that incorporate agarose polymers (pictured; Science 326, 1244-1247; 2009). Despite the fact that the inorganic phase contained this organic network, it was still single-crystalline in nature. Estroff and co-workers found that the internal surfaces that formed the inorganic-organic interface were a mixture of high- and lowenergy facets.

The polymer network could be removed after heating the crystals to $400^{\circ} \mathrm{C}$ for an hour, which left discrete cavities, but still a single crystal.

NEIL WITHERS

\section{CROSS-COUPLING}

\section{Stereochemistry by remote control}

The stereochemical lability of cycloalkylzinc reagents combined with a large difference in reactivity between epimers has been exploited to form a wide variety of interesting organic compounds with both high yields and diastereoselectivities.

\section{Frank Glorius}

$\mathrm{T}$ ransition-metal-catalysed crosscoupling - the reaction of organometallic reagents $(\mathrm{R}-\mathrm{M})$ with aryl halides and related compounds $\left(\mathrm{R}^{\prime}-\mathrm{X}\right)$ resulting in the formation of a new $\mathrm{C}-\mathrm{C}$ bond $\left(\mathrm{R}-\mathrm{R}^{\prime}\right)$ - has become a mature field and is an important tool for the formation of a wide array of compounds. The cross- coupling of two $s p^{2}$-hybridized carbon centres is well established and, more recently, the cross-coupling of $s p^{3}$-hybridized carbons has become an important and rewarding research area.

New stereocentres can be formed when secondary alkyl halides and/or alkyl-metal reagents are employed and several excellent stereoselective coupling methods have been developed ${ }^{1-4}$. The ability to achieve high levels of stereoselectivity is of the utmost importance for many applications, particularly in the field of life sciences where different enantiomers or diastereomers of a molecule very often have different biological activity. In addition, even when separation 